\title{
The ATLAS Liquid Argon Calorimeter: One year of LHC operation and future upgrade plans for HL-LHC
}

\author{
Peter W. Krieger, \\ on behalf of the ATLAS Liquid Argon Calorimeter Group
}

\begin{abstract}
An overview of the ATLAS liquid-argon calorimeter system is provided, along with a discussion of its operation and performance during the first year of LHC running. Upgrade planning related to the proposed high-luminosity upgrade of the LHC is also discussed, with an emphasis on the forward part of the calorimeter where the effects of the higher luminosity are a particular challenge.
\end{abstract}

\section{INTRODUCTION}

$\mathbf{T}$ HE ATLAS detector [1], illustrated in Figure 1, is one of the two general-purpose detectors built for the study of high-energy proton-proton ( $p-p)$ collisions at the CERN Large Hadron Collider (LHC) [2] and is designed to provide good discovery potential for the $\mathrm{TeV}$-scale physics to be explored there. It comprises three main sub-systems: an Inner Detector, which provides charged-particle tracking out to a pseudorapidity ${ }^{1},|\eta|$, of 2.5 , a Muon Spectrometer which can operate in standalone mode and provides coverage out to $|\eta|$ of 2.7 , and a calorimeter system which measures energies out to $|\eta|$ of 4.9. In the region of $|\eta|<1.7$ the hadronic calorimetry is provided by a steel and scintillating-tile calorimeter (Tile Calorimeter). The remainder of the calorimetry uses liquid argon as the active medium.

Data-taking with the full ATLAS detector began with global cosmic-ray running in 2008. A small amount of data was also recorded during the initial phase of LHC running with single beams, just prior to the incident of Sept. 19 and the subsequent shutdown for repairs. Data-taking with $\mathrm{p}-\mathrm{p}$ collisions began in November 2009 at $\sqrt{s}=900 \mathrm{GeV}$. After the year-end shutdown, LHC running resumed in March 2010, at $\sqrt{s}=7 \mathrm{TeV}$. Throughout 2010, the LHC operations team gained experience with the machine, providing incremental luminosity increases via, for example, higher bunch intensities, squeeze commissioning and increased numbers of bunches. This is visible in Figure 2 which shows the integrated luminosity profile for the 2010 p-p running; this was characterized by sufficiently low luminosity that pile-up was not a major issue for investigations of the detector performance. Since April 2011, the LHC has been regularly achieving worldrecord instantaneous luminosities with the most recent running having up to about $10 \mathrm{p}$-p interactions per bunch crossing. Studies of the detector performance under these conditions are

\footnotetext{
${ }^{1}$ In the ATLAS coordinate system, $\theta$ is the polar angle and $\phi$ is the azimuthal angle with respect to the beam axis. The pseudo-rapidity, $\eta$ is defined as $-\ln \tan (\theta / 2)$
}

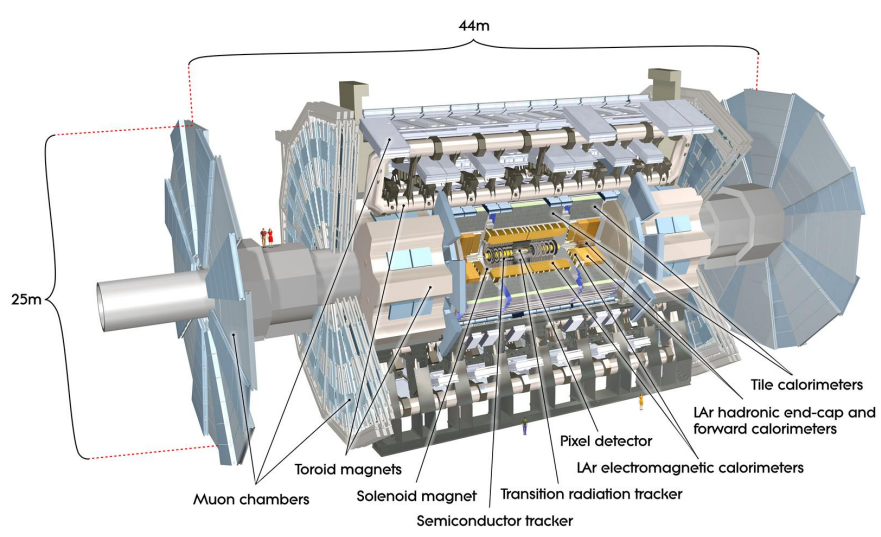

Fig. 1: The ATLAS Detector at the LHC.

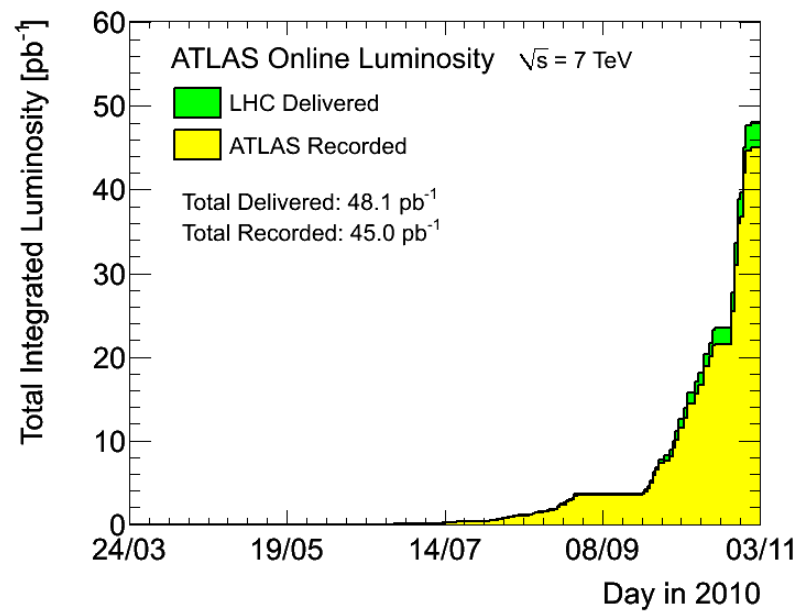

Fig. 2: ATLAS Cumulative integrated luminosity delivered and recorded for p-p collisions in 2010 .

now in progress. This paper will focus on the performance of the Liquid Argon (LAr) calorimeter during the 2009 and 2010 run periods.

\section{The ATLAS LiQuid ARgon CAlorimeter}

\section{A. Design and construction}

The LAr calorimeter system is illustrated in Figure 3 and some details of its sub-systems are provided in Table I. In the central region of the detector the barrel cryostat holds 


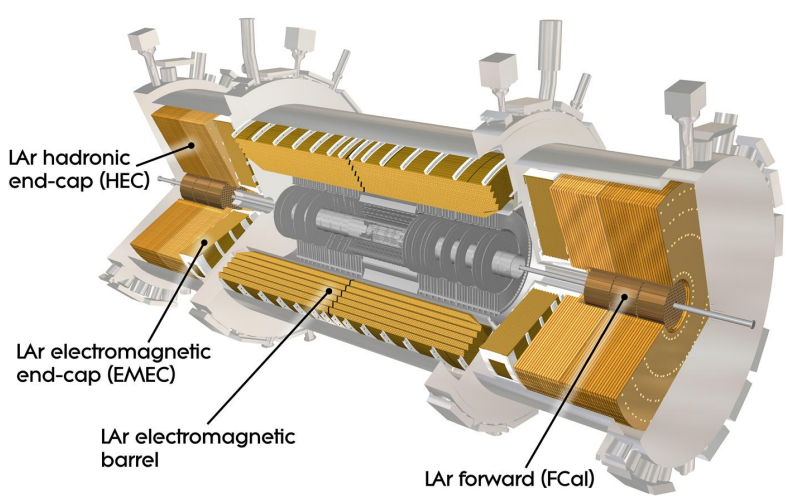

Fig. 3: The ATLAS Liquid Argon Calorimeter

\begin{tabular}{|l||c|c|r|}
\hline LAr system & Coverage & Absorber & Channels \\
\hline \hline EMB & $0<|\eta|<1.475$ & $\mathrm{~Pb}$ & 109568 \\
EMEC & $1.375<|\eta|<3.2$ & $\mathrm{~Pb}$ & 63744 \\
HEC & $1.5<|\eta|<3.2$ & $\mathrm{Cu}$ & 5632 \\
FCal & $3.1<|\eta|<4.9$ & $\mathrm{Cu} / \mathrm{W}$ & 3524 \\
\hline
\end{tabular}

TABLE I: LAr calorimeter sub-systems.

the LAr electromagnetic barrel (EMB) calorimeter, including a pre-sampler (PS), as well as the superconducting solenoid that provides the $2 \mathrm{~T}$ field for the Inner Detector. Separate cryostats hold the two endcap calorimeter systems, which comprise (on each side) the electromagnetic endcap calorimeter (EMEC), the hadronic endcap calorimeter (HEC) and the forward calorimeter ( $\mathrm{FCal})$.

The designs of the LAr sub-systems differ, according to both the performance requirements and the detector environment. The choice of LAr as the active medium was based on the need for radiation hardness and for a fast and uniform response, to allow as small a constant term as possible for, in particular, the resolution of large electromagnetic energy deposits. This requirement also drove the unusual design of the EMB calorimeter, a Pb-LAr system with accordion-shaped absorbers and electrodes, providing for full azimuthal coverage with no cracks. The EMB is segmented in depth into three layers (after the PS), the first being very finely segmented in order to provide for very good position resolution, which is also required for good resolution on the invariant mass of electromagnetic final states, as well as for $\gamma-\pi^{0}$ separation. An accordion structure was also chosen for the EMEC, though with some modifications due to the different geometry. It consists of two concentric wheels; the outer wheel covers up to $|\eta|$ of 2.5 with three longitudinal samplings and the inner wheel provides two samplings out to $|\eta|$ of 3.2. An endcap pre-sampler provides coverage out to $|\eta|$ of 1.8 .

The HEC has a more conventional parallel-plate structure, with copper absorbers. Each HEC is composed of two wheels consisting of 32 wedge-shaped modules, and two longitudinal samplings. The $8.5 \mathrm{~mm}$ gaps between the absorber plates hold a multi-gap electrode structure which subdivides the region into four $1.8 \mathrm{~mm}$ LAr drift regions.

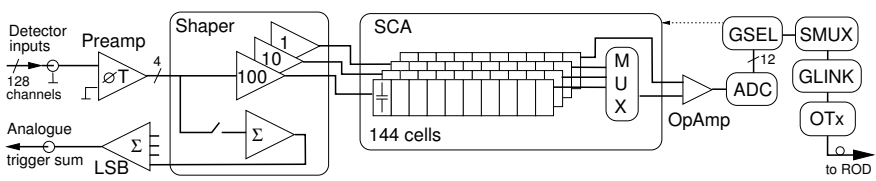

Fig. 4: Schematic of readout chain on the LAr FEBs.

The FCal consists of three disk-shaped modules, situated in the bore of the HEC. These are based on a novel design, relying on an array of tubular electrodes which form the very narrow LAr gaps $(269 \mu \mathrm{m}$ in the FCal1, the module closest to the interaction point) that are necessary for operation in the high-flux region at high $|\eta|$, in order to avoid signal degradation due to ion buildup in the gaps. The absorber material is copper for the FCall module, which is intended for EM calorimetry, and tungsten for the two hadronic modules, the FCal2 and the FCal3.

\section{B. LAr Calorimeter Readout \& Calibration}

Signals from the calorimeters are processed by front-end boards (FEBs) located in crates mounted directly on the cryogenic feedthroughs used to bring the signals out of the cryostat. The readout chain is illustrated in Figure 4. Signals are amplified and shaped to provide a fast, bipolar signal with equal-area positive and negative lobes, to prevent luminositydependent baseline shifts. There are four outputs from the shaper, corresponding to three gains (with ratios of $\sim 10$ ) and an input to the analog sum for the trigger tower to which the channel belongs. There are 58 LAr front-end crates (FECs), holding a total of 1524 FEBs, each reading out up to 128 cells. The FECs additionally contain calibration boards, trigger boards and controller boards used e.g. for FEB configuration and to receive and distribute timing signals. The number of readout channels in each LAr sub-system is given in Table I. Currently, the fractions of problematic channels are $0.06 \%$ for the EM calorimeter, $0.34 \%$ for the HEC and $0.11 \%$ for the FCal. The shaped signals are sampled every $25 \mathrm{~ns}$ according to a clock provided by the Trigger Timing and Control (TTC) system which delivers the timing signal to all ATLAS sub-systems; in normal LHC operation this is synchronized with the LHC clock. Samples are stored in a Switched Capacitor Array (SCA) analog pipeline until receipt of a Level-1 (L1) trigger signal, at which point they are read out and transmitted via optical fibres (one per FEB) to the back-end electronics located outside the detector cavern. Readout Drivers (RODs) processes the samples using a digital filtering algorithm, to calculate the signal amplitude (in MeV), timing, and a quality factor quantifying the agreement between the measured samples and the expected pulse shape. They also perform formatting and monitoring of the calorimeter signals before sending the data, via optical link, to the ATLAS data acquisition system. The digital-filtering procedure relies on calibration constants which are monitored regularly and updated when necessary, between ATLAS runs. The calibration scheme, which will be briefly described below, relies on good understanding of the pulse shape for each channel. For the EM calorimeters and the HEC, these are obtained from dedicated 
calibration runs. For the FCal, pulse shapes obtained from beam-test data are used.

Electronic calibration of the LAr system is performed using calibration boards installed in the same crates as the FEBs. These provide calibration signals of known amplitude, with a shape that approximates that of the expected physics pulse. For the EM calorimeters and the HEC, these pulses are injected into the readout chain close the calorimeter cell; in the case of the FCal, they are injected directly into the FEBs. Three types of standard calibration runs are taken regularly to determine the required channel-by-channel calibration constants: Pedestal runs provide the ADC pedestal and noise values; Ramp runs determine the response to signals of increasing amplitude, for determination of the electronic gains and Delay runs measure the response to calibration pulses with incremental timing offsets, for determination of pulse shapes.

For a given calorimeter cell, the energy at the electromagnetic scale is computed as:

$E_{\text {cell }}=F_{\mu \mathrm{A} \rightarrow \mathrm{MeV}} \cdot F_{\mathrm{DAC} \rightarrow \mu \mathrm{A}} \cdot \frac{1}{\frac{M_{\mathrm{phys}}}{M_{\mathrm{cali}}}} \cdot R\left[\sum_{j=1}^{N_{\text {samples }}} a_{j}\left(s_{j}-p\right)\right]$ where $F_{\mu \mathrm{A} \rightarrow \mathrm{MeV}}$ relates the ionization current in the detector to the deposited energy; these values are obtained from beamtest studies. $F_{\mathrm{DAC} \rightarrow \mu \mathrm{A}}$ describes the conversion between the digital-to-analog converter (DAC) setting on the calibration board and the amplitude of injected current pulse, while the factor of $\frac{M_{\text {phys }}}{M_{\text {cali }}}$ represents the ratio of the response to an ionization (physics) pulse to that of a calibration pulse of the same initial current. $R$ is the gain of the channel obtained from the Ramp run described above, $\left(s_{j}-p\right)$ represents the pedestalsubtracted samples, in ADC counts, and the $a_{j}$ are the optimalfiltering coefficients that are derived based on the known pulse shape and noise for each channel. A second set of optimalfiltering coefficients are used to obtain the timing. In normal running $N_{\text {samples }}=5$, but up to 32 samples can be taken; this is especially useful for pulse-shape studies. Calibrations are performed separately for each of the three gains used to cover the required dynamic range.

During the 2009 cosmic-ray commissioning period, the calibration stability was monitored [3] over a period of six months, over which the pedestal stability was better than 0.03 ADC counts and the gain stability was better than $0.1 \%$. During normal operation Pedestal and Ramp runs are performed at least four times per week, while Delay runs are taken at least once per week. A dedicated team analyzes the results of each set of calibration runs and updates the database as needed.

An overview of the performance of the LAr readout electronics can be found in reference [4].

\section{Detector Operation}

The LAr calorimeters have been fully instrumented and operating at nominal high voltage since 2006, when data from cosmic-ray interactions were first recorded as part of the LAr calorimeter commissioning exercise. ATLAS global cosmic-ray running began in 2008 and was an important part of the commissioning of the detector and the Trigger and Data Acquisition (TDAQ) system. In September 2008 first beams were injected into the LHC. Single circulating beams were established and beam-splash events, in which a single proton bunch was directed onto a collimator upstream of the detector, were used to illuminate the detector. These cosmicray and beam-splash data have been used for a number of important commissioning studies, particularly those related to understanding of the pulse shapes [3], [5]. A well-publicized accident on Sept. 19, 2008 resulted in the shutdown of the LHC prior to first collisions. Beams were re-introduced into the LHC in November 2009 and first collisions at $\sqrt{s}=$ $900 \mathrm{GeV}$ followed quickly thereafter. Since March 2010 the LHC has been running at an energy of $3.5 \mathrm{TeV} /$ beam. This is planned to continue through to the end of 2012.

This section briefly reviews the operational status of the LAr calorimeter. Following a discussion of some of the problems experienced, the data-quality (DQ) infrastructure and procedures are discussed.

\section{A. Hardware problems in 2010}

The performance of the detector has been very good throughout the commissioning and early data-taking. Nevertheless, some problems have been encountered. The most serious of these have been related to the low-voltage power supplies (LVPS), actually DC-DC converters that provide the various low voltages required by the front-end electronics, and to the optical transmitters (OTx), one per FEB, that are used to transmit the data to the back-end system. Because the front-end electronics and the LVPS are on-detector, in the experimental cavern, access is not possible except in periods of prolonged shutdown. So reliability of this equipment is critical. The LVPS were a source of early problems, with failures both during long-term burn-in tests, and after installation on the detector. They are designed with some redundancy; while there were several supplies on-detector that had failures which resulted in a loss of this redundancy, no full failures have occurred since the start of LHC collisions, so data-taking has not been affected. However, uncertainty about their future reliability has led to the design and manufacture of new supplies. These have all been received and will be extensively tested before being installed during the long LHC shutdown, now anticipated for 2013.

Failures of the OTx devices began in early 2008 and continued to develop at an increasing rate through to the fall of 2009 after which the problem slowed, presumably as those OTx that were prone to failure succumbed. This is illustrated in Figure 5(a) which shows the cumulative distribution of failures as a function of time since January 2008. This cause of this problem was extensively investigated. While no definitive explanation was found, in situ investigations of the on-detector OTx showed that those prone to failure had narrower than expected optical spectra, as shown in Figure 5(b). This allowed for a robust identification of problematic OTx still on the detector. Over the 2010-11 shutdown all FEBs with OTx that had failed, or which were identified as likely to fail, were replaced by spares having OTx with nominal optical properties. There have been no failures in 2011.

The other major hardware intervention undertaken over the 2010-11 shutdown was the repositioning of the end- 


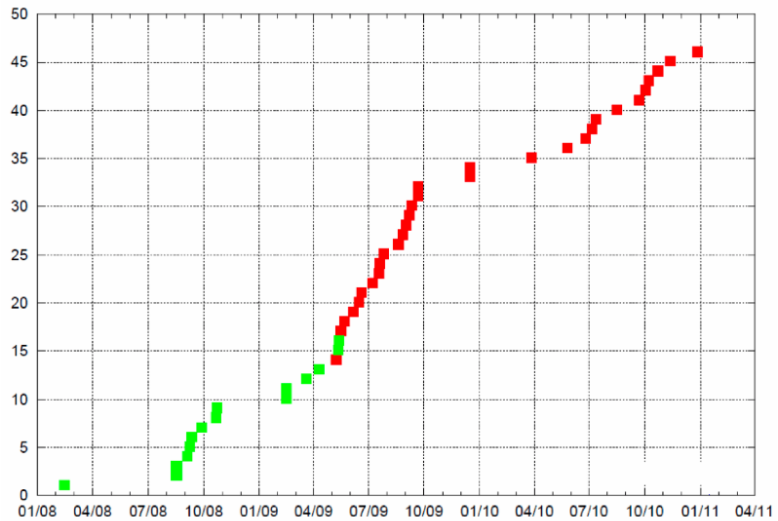

(a)

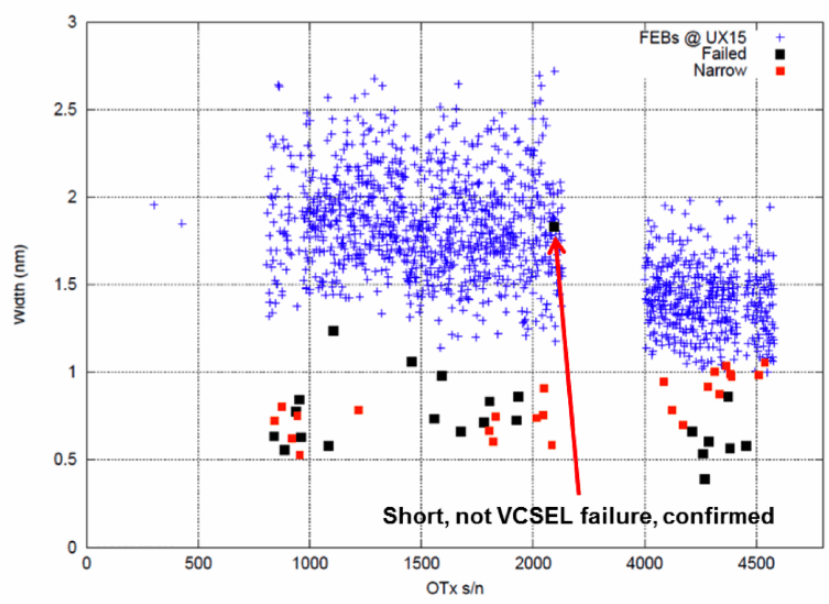

(b)

Fig. 5: LAr optical transmitter (OTx) failures: (a) shows The cumulative number as a function of time since January 2008. The green markers indicate OTx on FEBs that were replaced during a shutdown at the beginning of 2009 and the red markers indicate the failures since then. (b) shows the width of the optical spectrum plotted against the serial number for all devices installed on the detector for the 2010 run. The black markers indicate those that have failed since September 2009; the red markers indicate OTx on FEBs that were preemptively replaced during the 2010-11 shutdown.

cap cryostats. Analysis of the FCal position relative to the beamspot showed that on each side of ATLAS the FCal was sitting about $2 \mathrm{~mm}$ low. Additional studies of the EMEC and its position relative to the Inner Detector led to the conclusion that the small vertical shift was a global endcap effect, not specific to the FCal. When the calorimeter endcaps were moved back into position after the maintenance and repair work done during the shutdown, they were positioned approximately $2 \mathrm{~mm}$ higher than their pre-shutdown positions.

\section{B. Detector and Data Quality Monitoring}

The status of the LAr calorimeter is available via the Detector Control System (DCS), which monitors and logs hardware parameters relevant to the front and back-end electronics, the high-voltage (HV) system and the cryogenics (e.g. the LAr temperature and purity). This information is available to the control-room shifters responsible for operation of the detector, and to offline shifters responsible for data-quality assessments. Luminosity blocks (corresponding to about 2 minutes of data in the 2010 running) are flagged as bad for any sub-system that has e.g. a non-nominal $\mathrm{HV}$ value due, for example to an HV trip. The physics data coming from the detector is also continuously monitored via online histograms that are available to the shifter as well as to automated online monitoring tools and offline data quality experts. These can be used, for example, to identify channels or regions that are sporadically noisy, or regions where coverage changes due to problems that appear during running. Luminosity blocks are also marked as bad if, for example, there is evidence of noise bursts in one of the LAr sub-systems, or if there are FEB errors resulting from data corruption.

ATLAS data reconstruction is based on an initial 36 hour "calibration loop": a preliminary DQ assessment is done online by the Data Quality Monitoring software, based on examination of a large number of online histograms. Results from the reconstruction of a dedicated express data stream, along with information from DCS is used by offline data-quality shifters to refine the DQ assessment and to flag good luminosity blocks within the 36-hour period, after which bulk reconstruction of the data begins. Data-quality flags are collected from all ATLAS sub-systems to define the good run lists (GRLs) that are used by the physics groups for data analysis.

\section{Performance Studies With Data}

The primary function of the ATLAS calorimeter is to measure the energy of high- $p_{\mathrm{T}}$ jets, photons and electrons, as well as the transverse energy $\left(E_{\mathrm{T}}\right)$ balance in the event. Missing transverse energy $\left(E_{\mathrm{T}}^{\text {miss }}\right)$ is a signature of the production of massive particles that escape detection, such as are predicted by numerous theories of physics beyond the Standard Model.

An important part of any modern particle physics experiment is a detailed simulation of the detector response; comparisons of distributions from data analysis with the predictions Monte Carlo simulations play an important role in evaluations of the detector performance. Discrepancies between data and Monte Carlo can arise either due to the presence of new (or unmodeled) physics, or to inadequacies in the detector simulation. Good agreement between data and Monte Carlo for the response to well understood SM processes is necessary before any claim of physics beyond the SM can be taken seriously. Numerous performance studies of the ATLAS detector, and the LAr Calorimeter have been undertaken using the $\mathrm{p}-\mathrm{p}$ collision data sample from 2009-10. Some of these, related to the refinements of the EM energy scale, signal timing and performance for missing energy are discussed briefly below.

\section{A. EM energy scale uniformity}

The electromagnetic energy scales for the LAr calorimeters and the hadronic calibration schemes used by ATLAS are based on extensive standalone and combined beam-testing of the different sub-systems. All energy measurements start from 


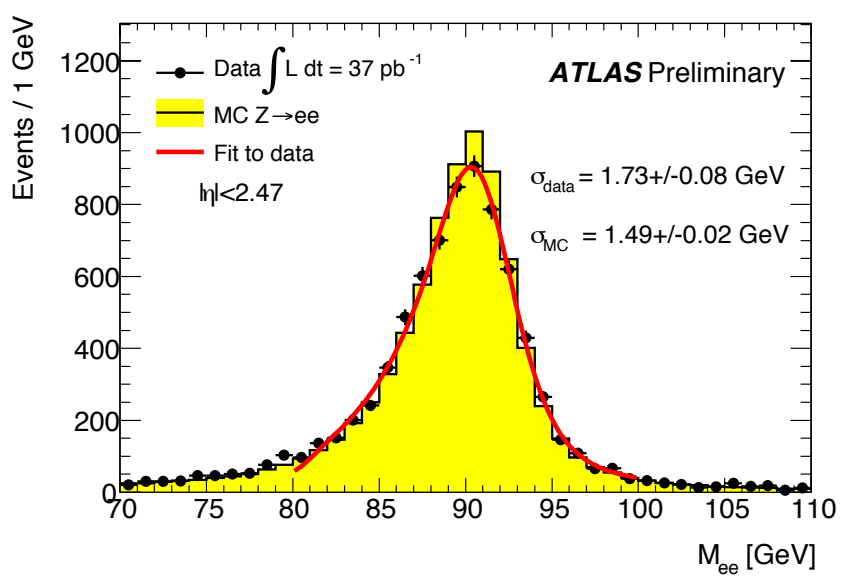

Fig. 6: $Z \rightarrow e e$ di-electron invariant mass distributions for electrons in the central region, $|\eta|<2.47$.

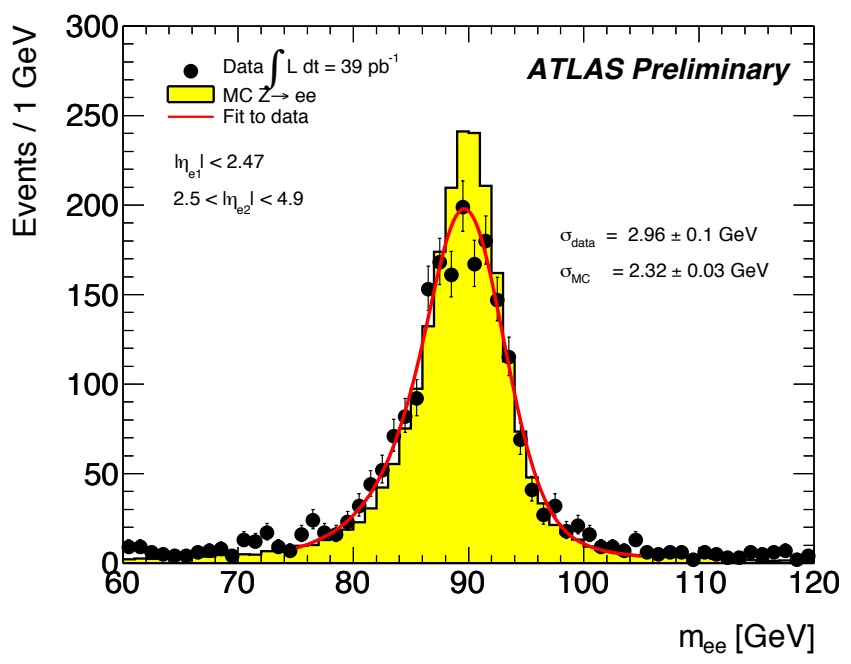

Fig. 7: $Z \rightarrow e e$ invariant mass distributions for central-forward opposite sign electrons pairs: e.g. one electron satisfying $|\eta|<2.47$, and other with $|\eta|>2.5$.

the cell-level EM scale energies from the calorimeter. The scale and uniformity of the EM response have been checked over the full acceptance of the LAr calorimeter by using the 2010 data to reconstruct well-understood resonances decaying to electromagnetic final states.

The response to high- $p_{\mathrm{T}}$ electrons has been investigated using $Z \rightarrow e e$ decays. In different regions of $\eta$ the invariant mass distribution of opposite-sign electron candidates having $E_{\mathrm{T}}>20 \mathrm{GeV}$ was examined in a window around the mass of the $Z$ boson. Corrections to the default energy scale were defined by requiring that this distribution follow the well known $Z$ line shape. These were of order $1 \%$ in the barrel-region of the detector and $2 \%$ in the endcap regions, out to the edge of the Inner Detector acceptance at $|\eta|$ of about 2.5. Using the re-calibrated energy scale for this central region, $Z \rightarrow$ ee decays were also used to study the EM scale in the region $|\eta|>2.5$ where, due to the absence of tracking, electrons are identified based on cluster shapes in the

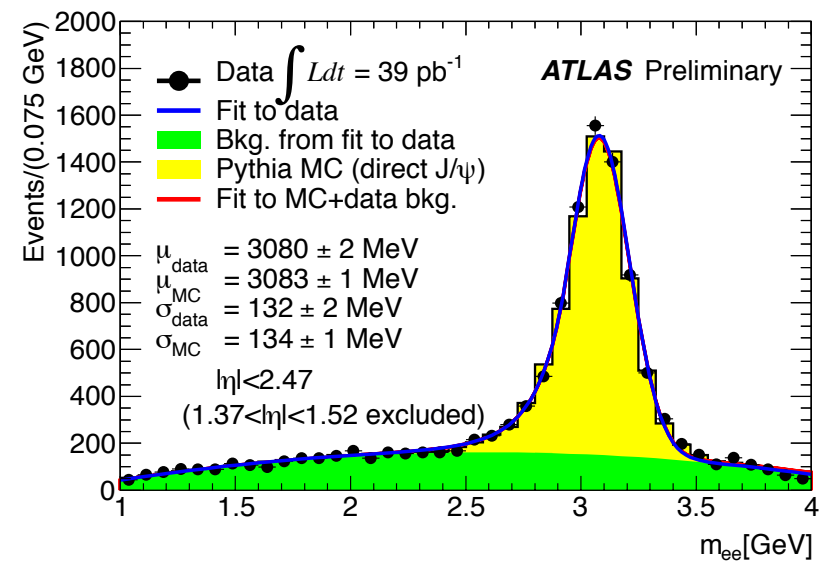

Fig. 8: Data and Monte Carlo di-electron invariant mass distribution.

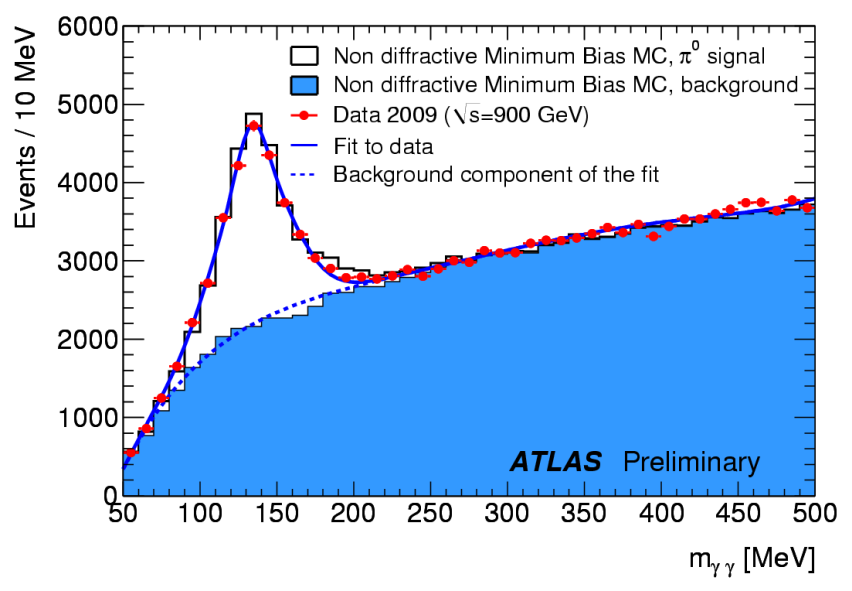

Fig. 9: Di-photon invariant mass distribution.

calorimeter. The invariant mass distribution of opposite-sign central-forward electron pairs is compared to that expected for the $Z$ and energy-scale corrections for the forward region are derived in bins of $\eta$. These were of order $1 \%$ in the inner wheel of the EMEC and about 4\% in the FCal. The invariant mass distributions of the central-central and central-forward combinations are shown in Figures 6 and 7, respectively, after application of these energy-scale corrections. Good agreement with the simulation is observed.

The reconstruction of lower $p_{\mathrm{T}}$ electrons has been investigated by looking at the decay $J / \Psi \rightarrow e e$, with the $\eta$-dependent energy-scale corrections from the $Z \rightarrow e e$ analysis applied. The result of this analysis is presented in Figure 8 which shows excellent agreement between data and Monte Carlo. A similar analysis was previously done using photons from $\pi^{0}$ decays [6]. The invariant mass of selected photon pairs in the range $|\eta|<2.5$ is shown in Figure 9, based on the analysis of $900 \mathrm{GeV}$ p-p collision data. The agreement between data and Monte Carlo is good. However, this analysis predates the availability of the energy scale corrections described above. An analysis of the data / MC agreement in bins of $\eta$ shows differences consistent with the size of the corrections derived 

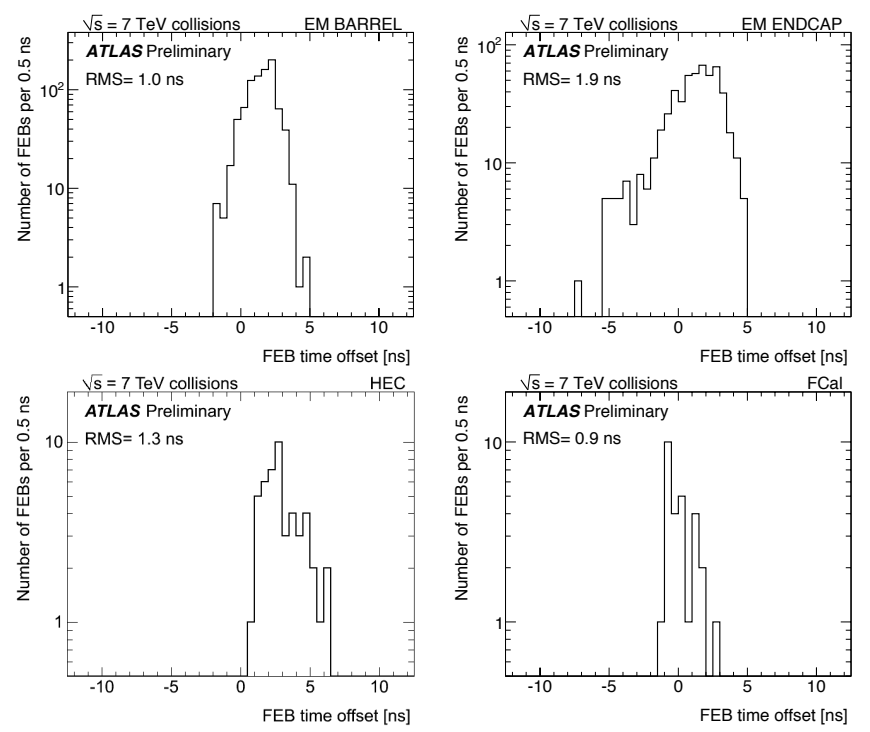

Fig. 10: Distributions of FEB average times for the EMB, EMEC, HEC and FCal, in April 2010.

from the $Z \rightarrow e e$ analysis, that is, about $1 \%$ in the barrel region and $2 \%$ in the endcap region out to $|\eta|$ of 2.5 . The overall uncertainty on those measurements is about $2 \%$. The $\phi$ uniformity of the reconstructed mass is better than $0.7 \%$.

\section{B. Timing studies}

Coarse adjustment of the LAr calorimeter timing can be achieved using configurable delays on the FEBs. The distributions of the average timing for the FEBs from all LAr sub-systems, near the start of the 2010 run, are shown in Figure 10. After correcting for the FEB delays obtained from the collision data, the RMS of the timing is $\sim 1 \mathrm{~ns}$ for all LAr sub-systems. Finer adjustments can also be made channel-bychannel, via the optimal-filtering coefficients used to extract the signal timing. The ultimate goal is a timing resolution of around $100 \mathrm{ps}$.

\section{Performance for missing transverse energy}

Proper determination of the event missing transverse energy relies on the full calorimeter. However, the performance of the LAr system alone was investigated using randomly triggered events during the 2009 cosmic-ray run periods. In such events, no real $E_{\mathrm{T}}^{\mathrm{miss}}$ is expected; the width of the distribution obtained from such an analysis is related to the electronic noise. Figure 11 shows the $E_{\mathrm{T}}^{\mathrm{miss}}$ distributions from both a simple cell-based calculation using all LAr cells with $|E|>2 \sigma_{\text {noise }}$ and for a calculation based on topological clusters [7], which provide for better noise suppression. Both distributions are compared with the predictions of a simple Gaussian noise model. The result from the topological clustering provides better resolution due to superior noise suppression, but somewhat poorer agreement with the simple Gaussian noise model, due to the presence of some channels with larger than average noise.

The results of the ATLAS $E_{\mathrm{T}}^{\text {miss }}$ calculation using the full calorimeter, applied to minimum-bias events from the $7 \mathrm{TeV}$

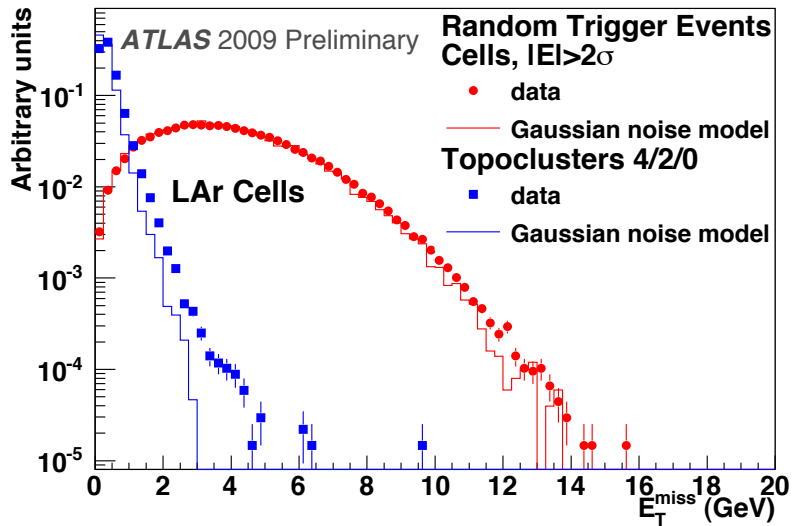

Fig. 11: $E_{\mathrm{T}}^{\text {miss }}$ distribution from randomly-triggered events using two techniques, a simple cell-based method and one relying on a topological clustering method.

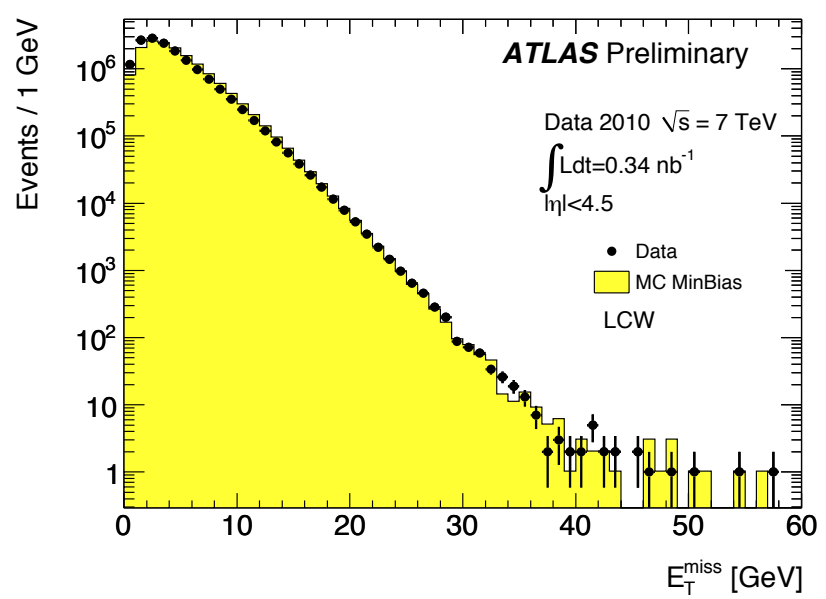

Fig. 12: $E_{\mathrm{T}}^{\text {miss }}$ distribution from $7 \mathrm{TeV}$ p-p minimum bias events taken in early 2010 . The points represent the data while the histogram shows the distribution expected from the Monte Carlo simulation.

p-p collision data sample are shown in Figure 12 [8] and shows excellent agreement with predictions of the Monte Carlo simulation.

\section{LAR HIGH-LUMINOSITY UPGRADE PLANS}

The future planning for the LHC includes a luminosity upgrade starting sometime after 2020 with the goal of reaching a peak luminosity of $5 \times 10^{34} \mathrm{~cm}^{-2} \mathrm{~s}^{-1}$, with leveling. The aim is to collect $250 \mathrm{fb}^{-1} /$ year and a total of $3000 \mathrm{fb}^{-1}$, a factor of 10 more than is planned for the first decade of LHC operation.

Such a high luminosity poses several challenges to ATLAS as a whole, and will, for instance, require replacement of the Inner Detector. For the LAr calorimeter, there are several issues, including radiation hardness of the front-end electronics. The current FEB architecture was qualified for 10 years of LHC operation, with a limited number of spares and is not considered appropriate for HL-LHC running. While there is a significant effort in progress to develop a new readout 


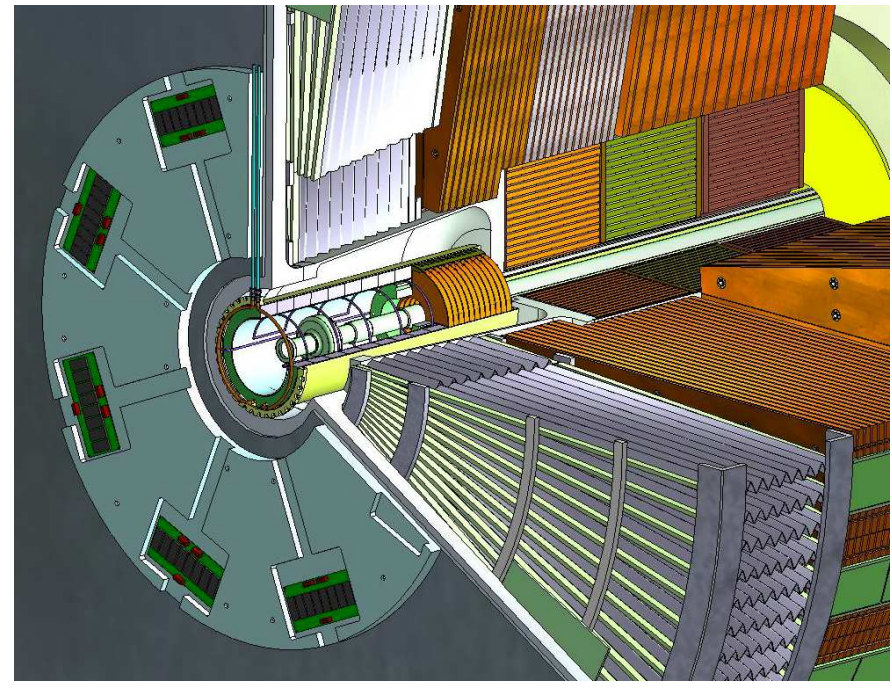

(a)

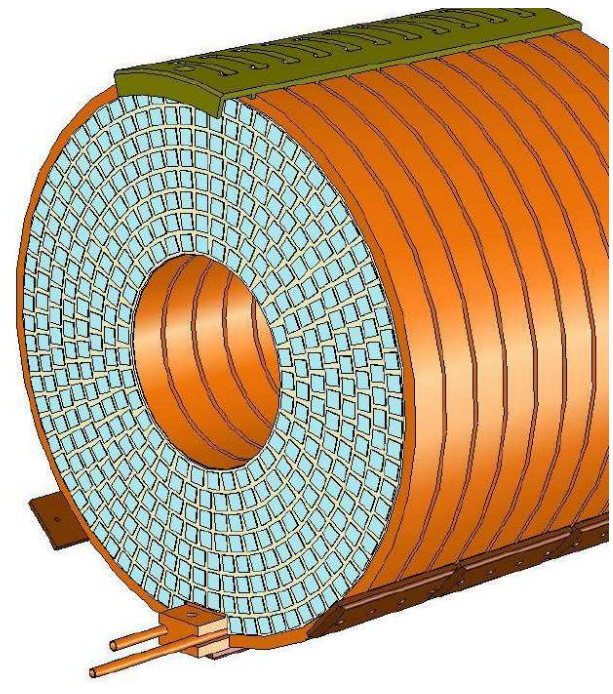

(b)

Fig. 13: The Mini-FCal: (a) shows the placement of the Mini-FCal inside the forward cone of the LAr endcap cryostat support tube, in front of the FCal. (b) shows the proposed design, with copper absorber plates and diamond sensor layers. The first copper plate has been omitted to permit a view of the sensor plane. Also visible at the bottom are the ends of the cooling loop.

architecture, the focus here is on those upgrades requiring a hardware intervention on the calorimeters themselves. Since the front-end electronics are dominantly on the FEBs, the intervention required for installation of upgraded boards should not pose any special challenge. However, the HEC uses cold GaAs preamplifiers located within the endcap cryostats, along the outer periphery of the HEC wheels. These are certified for $1000 \mathrm{fb}^{-1}$ at the LHC, with a safely factor of 10 . A correct understanding of whether they will continue to operate properly at the HL-LHC will rely on improved understanding of the radiation levels in the region they occupy and an estimate of the safety factor required. Predictions coming from simulations will be complemented by measurements of the cavern backgrounds at different positions in and around the detector, with the goal of understanding the uncertainty on the simulation predictions by the end of 2012 .

Replacement of the HEC cold preamplifiers would require a major effort, since the cryostats (which are welded shut) would need to be opened and the HEC wheels extracted. To complicate matters further, given the anticipated time constraints, this would almost certainly have to be done in the rather cramped confines of the experimental cavern, where other work will also need to take place.

The other main issue for the LAr calorimeter is the forward region. The FCal was designed with very narrow LAr gaps in order to allow it to operate in the harsh region it occupies near the ATLAS beampipe. These are necessary in order to avoid positive ion buildup that would distort the electric field in the gap, changing the response in a luminosity-dependent way that would also vary with the radius from the beam line. There is potentially also an associated problem related to beam-heating of the detector due to the very high ionization load, which could cause localized boiling of the liquid argon. Finally, the HV distribution to the FCal electrodes is via either $1 \mathrm{M} \Omega$ or
$2 \mathrm{M} \Omega$ resistors on 'summing boards' mounted on the rear of the HEC, inside the cryostat. With the increased current drawn by the FCal at very high luminosity, the voltage drop across these resistors becomes large relative to the applied voltage; this effect also varies with the radial position of the cells.

Because of activation issues, modifications to the existing FCal will likely not be possible. There are currently two solutions proposed to maintain calorimeter performance in this difficult region. This first is the replacement of the $\mathrm{FCal}$ with a similar device built with even narrower LAr gaps $(100 \mu \mathrm{m}$ in the FCal1), cooling loops to ameliorate any beam heating problems, and a new HV distribution system. There are two scenarios for the installation of this 'sFCal', both of which require opening parts of the endcap cryostat: in the case where HEC cold electronics need replacement, the FCal would be replaced with the sFCal and new summing boards, with lower HV protection resistors, would replace those that are currently mounted on back of the HEC. If the HEC electronics do not need replacement, the warm cover could be removed and the FCal, which is assembled within an aluminum tube that forms part of the endcap mechanical support structure, could be extracted without removal of the cold cover. This would mean abandoning the existing FCal summing boards and installing a new system, possibly behind the un-instrumented plug that sits behind the FCal3 module.

There are currently a number of $R \& D$ projects underway to examine the behaviour of the three endcap calorimeter systems under the high fluences that will be present at the HL-LHC. These include high-intensity beam tests (using the $70 \mathrm{GeV}$ proton synchrotron in Protvino, Russia) of EMEC, HEC and FCal cells, including one with the proposed $100 \mu \mathrm{m}$ gaps, as well as dedicated measurements of LAr properties such as ion mobility that currently contribute to uncertainties on projected FCal performance at high luminosities. 
The opening of any part of the endcap cryostat may be deemed undesirable in the case where investigations conclude that the HEC cold electronics need not be replaced. A second, somewhat less invasive solution to the problem has thus been developed, which involves the installation of a new warm calorimeter in front of the existing FCal. This would absorb a large portion of the flux that would otherwise reach the innermost part of the $\mathrm{FCal}$, reducing it to a level at which the existing FCal can operate normally. Beam-heating, ionbuildup and voltage drop across the protection resistors are all potentially addressed by this solution. The device, for now referred to as the Mini-FCal, has a transverse size that is constrained by the dimensions of the existing cryostat support structure into which it must be installed, so it must be both compact and radiation hard. The current proposal is a cylindrical sampling calorimeter with a parallel-plate structure using copper absorbers and diamond sensors as the active layer. This is illustrated in Figure 13, which shows the MiniFCal design and its location within the endcap cryostat.

A crucial aspect of the Mini-FCal design is an understanding of the radiation tolerance of the diamond sensors proposed for the active layers. Ten years of running at the HL-LHC is assumed to correspond to about $2 \times 10^{17}$ neutrons $/ \mathrm{cm}^{2}$, which exceeds the dose to which any previous diamond detectors have been tested. Both polycrystalline and singlecrystal Chemical Vapour Deposition diamond detectors have been investigated by the RD42 collaboration at CERN at fluences up to about $2 \times 10^{16}$ particles $/ \mathrm{cm}^{2}$ [9]. In those tests, the detector response was reduced to $\approx 20 \%$ of the initial value, consistent with an exponential deterioration. In order to study the response at higher dose, two grades of polycrystalline chemical vapour deposition (pCVD) diamond detectors ${ }^{2}$ with two different metalizations were continuously monitored during irradiation with $500 \mathrm{MeV}$ protons at TRIUMF [10] to a fluence of $2.25 \times 10^{17}$ particles $/ \mathrm{cm}^{2}$. The response of the four detectors as a function of the integrated particle fluence is shown in Figure 14. After the full exposure the response is reduced to about $5 \%$.

It is likely that the Mini-FCal preamplifiers will need to be located not too far from the device itself (see for instance, Figure 13(a) where they are mounted on the outer wall of the endcap cryostat). However the plan for the front-end electronics is to use slightly modified LAr FEBs located in the existing FCal crate, which is now only half populated. This limits the number of channels to under about $10^{3}$. In the current design, each Mini-FCal would consist of 12 absorber layers and 11 sensor planes holding a total of $\sim 8 \mathrm{~K}$ diamond sensors. Various tiling patterns for the sensors have been simulated, in order to find an arrangement that minimizes both gaps and overlaps. In order to keep the number of readout channels at an acceptable level, individual sensors will be ganged together to form readout cells; since the radiation dose will be different at different depths and radii within the device, care must be taken to gang together cells that have similar exposures, in order to simplify the calibration scheme that

\footnotetext{
${ }^{2}$ Diamond Detectors Ltd, 16 FleetBridge Business Centre, Upton Road, Poole UK,BH17 7AF
}

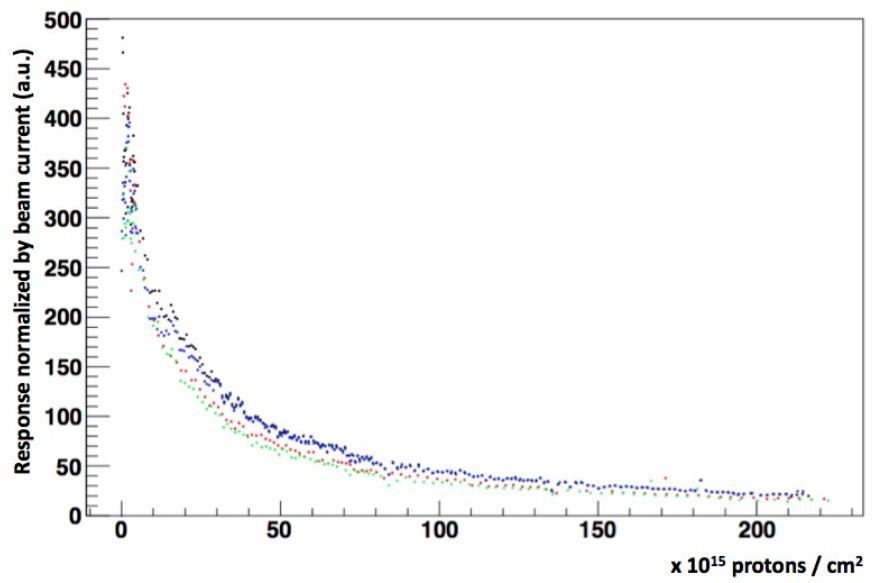

Fig. 14: Response vs. exposure for four diamond detectors irradiated at TRIUMF.

will be needed to account for the differential degradation of the response. A number of sensor-tiling and ganging scenarios have been simulated, using the measured damage curves as input. This work is on-going.

\section{ACKNOWLEDGMENT}

The ATLAS Liquid Argon calorimeter has been operating successfully for almost half a decade, starting with cosmicray data-taking in 2006, for p-p collisions starting in 2009 and for $\mathrm{Pb}-\mathrm{Pb}$ collisions in late 2010. Despite some problems encountered along the way, the performance of the calorimeter has been excellent. This is the result of the dedicated work of the large team of people involved in the R\&D, construction and commissioning phases, as well as to the efforts of a talented and diligent operations team, who maintain the detector hardware, perform and monitor the calibrations, and pursue the technical studies necessary to ensure that high data-taking efficiency and good data quality are maintained.

\section{REFERENCES}

[1] G. Aad et al., The ATLAS Experiment at the CERN Large Hadron Collider, JINST 3 (2008) S08003.

[2] L. Evans and P. Bryant eds., LHC machine, JINST 3 (2008) S08001.

[3] G. Aad et al., Readiness of the ATLAS Liquid Argon Calorimeter for LHC Collisions, Eur. Phys. J. C 70 (2010) 723.

[4] H. Abreu et al, Performance of the electronic readout of the ATLAS liquid argon calorimeters, JINST 5 (2010) P09003.

[5] G. Aad et al., Drift Time Measurement in the ATLAS Liquid Argon Electromagnetic Calorimeter, Eur. Phys. J. C 70 (2010) 755.

[6] ATLAS Collaboration, Performance of the ATLAS electromagnetic calorimeter for $\pi^{0} \rightarrow \gamma \gamma$ and $\eta \rightarrow \gamma \gamma$ events. ATLAS-CONF-2010006 (2010).

[7] T. Barillari, et al., Local Hadronic Calibration, ATL-LARG-PUB-2009001-2, (2010).

[8] ATLAS Collaboration, Performance of the Missing Transverse Energy Reconstruction and Calibration in Proton-Proton Collisions at a Centerof-Mass Energy of $\sqrt{s}=7 \mathrm{TeV}$ with the ATLAS Detector. ATLASCONF-2010-057, (2010).

[9] M. Barbero et al., (RD42 Collaboration), Development of Diamond Tracking Detectors for High Luminosity Experiments at the LHC, CERN/LHCC 2008-005.

[10] D. Axen et al., Diamond Irradiation Tests at TRIUMF, submitted to JINST, March 2011. 